\title{
Tet2-mediated clonal hematopoiesis in nonconditioned mice accelerates age-associated cardiac dysfunction
}

\author{
Ying Wang, ${ }^{1,2}$ Soichi Sano, ${ }^{1}$ Yoshimitsu Yura, ${ }^{1}$ Zhonghe Ke, ${ }^{3}$ Miho Sano, ${ }^{1}$ Kosei Oshima, ${ }^{4}$ \\ Hayato Ogawa, ${ }^{1}$ Keita Horitani, ${ }^{1}$ Kyung-Duk Min, ${ }^{1}$ Emiri Miura-Yura, ${ }^{1}$ Anupreet Kour, ${ }^{1}$ Megan A. Evans, ${ }^{1}$ \\ Maria A. Zuriaga, ${ }^{5}$ Karen K. Hirschi, ${ }^{1}$ Jose J. Fuster, ${ }^{5}$ Eric M. Pietras, ${ }^{3}$ Kenneth Walsh ${ }^{1}$ \\ ${ }^{1}$ Hematovascular Biology Center, Robert M. Berne Cardiovascular Research Center, University of Virginia School of \\ Medicine, Charlottesville, Virginia, USA. ²Department of Cardiology, Xinqiao Hospital, Army Medical University, Chongqing, \\ China. ${ }^{3}$ Division of Hematology, Department of Medicine, University of Colorado Anschutz Medical Campus, Aurora, \\ Colorado, USA. ${ }^{4}$ Molecular Cardiology/Whitaker Cardiovascular Institute, Boston University School of Medicine, Boston, \\ Massachusetts, USA. ${ }^{5}$ Centro Nacional de Investigaciones Cardiovasculares (CNIC), Madrid, Spain
}

Clonal hematopoiesis of indeterminate potential is prevalent in elderly individuals and associated with increased risks of all-cause mortality and cardiovascular disease. However, mouse models to study the dynamics of clonal hematopoiesis and its consequences on the cardiovascular system under homeostatic conditions are lacking. We developed a model of clonal hematopoiesis using adoptive transfer of unfractionated ten-eleven translocation 2-mutant (Tet2-mutant) bone marrow cells into nonirradiated mice. Consistent with age-related clonal hematopoiesis observed in humans, these mice displayed a progressive expansion of Tet2-deficient cells in multiple hematopoietic stem and progenitor cell fractions and blood cell lineages. The expansion of the Tet2-mutant fraction was also observed in bone marrow-derived CCR2 ${ }^{+}$myeloid cell populations within the heart, but there was a negligible impact on the yolk sac-derived CCR2- cardiac-resident macrophage population. Transcriptome profiling revealed an enhanced inflammatory signature in the donor-derived macrophages isolated from the heart. Mice receiving Tet2-deficient bone marrow cells spontaneously developed age-related cardiac dysfunction characterized by greater hypertrophy and fibrosis. Altogether, we show that Tet2-mediated hematopoiesis contributes to cardiac dysfunction in a nonconditioned setting that faithfully models human clonal hematopoiesis in unperturbed bone marrow. Our data support clinical findings that clonal hematopoiesis per se may contribute to diminished health span.

Authorship note: YW, SS, and YY contributed equally to this work.

Conflict of interest: The authors have declared that no conflict of interest exists.

Copyright: (c) 2020, American Society for Clinical Investigation.

Submitted: November 22, 2019

Accepted: February 26, 2020

Published: March 10, 2020

Reference information: JCI Insight 2020;5(6):e135204.

https://doi.org/10.1172/jci.

insight.135204.

\section{Introduction}

Clonal hematopoiesis can result from the acquisition of somatic mutations in preleukemic driver genes in hematopoietic stem cells (HSCs). Of these, mutations in the epigenetic regulators DNA methyltransferase 3 alpha (DNMT3A) and ten-eleven translocation 2 (TET2) are frequently observed in individuals with clonal hematopoiesis in the absence of cytopenias and dysplastic hematopoiesis, a condition that has been referred to as clonal hematopoiesis of indeterminate potential or age-related clonal hematopoiesis $(1,2)$ These mutations are thought to provide HSCs a competitive advantage within the bone marrow niche that leads to their clonal expansion. Clonal hematopoiesis is prevalent in the elderly, presumably because of the age-dependent accumulation of somatic mutations in HSCs and/or the degradation of the bone marrow niche that may favor the expansion of the mutant clones. As these clones expand, they increasingly give rise to progeny blood cells that harbor the mutation. Although clonal hematopoiesis generally does not lead to changes in total blood cell numbers, the leukocytes derived from these mutant clones can exhibit altered inflammatory properties (3-9).

A number of recent large cohort studies have associated clonal hematopoiesis with an increase in all-cause mortality (9-13). Clonal hematopoiesis markedly increases an individual's risk of developing a hematological malignancy, presumably because of the increased probability of acquiring additional driver mutations that lead to subsequent clonal expansions $(12,13)$. However, this cannot entirely explain the 
increase in mortality because of the relatively low incidence of hematological disease in the general population. In this regard, studies have associated clonal hematopoiesis with increased risks of coronary heart disease, stroke, and early-onset myocardial infarction $(7,13)$. Recently, clonal hematopoiesis resulting from inactivating mutations in DNMT3A or TET2 has been associated with future risk of cardiovascular disease and poor prognosis in patients with ischemic heart failure and in patients who have undergone treatment for aortic valve stenosis $(14,15)$. Experiments in mice suggest that clonal hematopoiesis might be causal for cardiovascular disease $(16,17)$. For example, it has been shown that inactivating mutations in Tet 2 or Dnmt3a can enhance cardiovascular tissue damage in response to injury $(3-5,7)$. These disease models include hyperlipidemia, permanent ligation of the left anterior descending artery, transverse aortic constriction, and the continuous infusion of pressor doses of angiotensin II. In the case of Tet2, it has been reported that homozygous mutations undergo more rapid expansion than heterozygous mutations and exhibit greater pathological phenotypes, indicative of a dose-dependent relationship $(3,5)$. However, to date, no model of clonal hematopoiesis has been shown to be sufficient to promote damage to cardiovascular tissues in the absence of an external injury.

In prior studies, we modeled the expansion of mutant HSC clones by performing competitive bone marrow transplantation (BMT) studies in mice that were subjected to a lethal dose of radiation (3-6). These competitive BMT models serve to minimize the confounding effects that these driver gene mutations exert in animal models, including elevated immune cell levels and systemic tissue inflammation, which are generally not features observed in individuals with clonal hematopoiesis. Regardless, these experimental systems have significant drawbacks in modeling human clonal hematopoiesis because of the systemic effects of irradiation on cardiovascular tissues (18), damage to the bone marrow niche, (19) and lineage bias due to the differentiation of a small fraction of dominant HSC clones (20). In view of these issues, we investigated a model of murine clonal hematopoiesis that involves the adoptive transfer of unfractionated bone marrow cells to mice that have not been preconditioned by a myeloablative strategy. This system allowed us to explore the process of Tet 2 mutation expansion in multiple hematopoietic cell populations and assess how they affect the heart under homeostatic conditions. Using this model, we elaborate the expansion kinetics of Tet2-deficient cells in bone marrow, blood, and tissues. We find that Tet2-mediated clonal hematopoiesis alters the phenotype of cardiac macrophages and promotes age-dependent cardiomyopathy in the absence of preexisting cardiovascular injury.

\section{Results}

Engraftment following myeloablation versus nonconditioned adoptive transfer. Our previous studies of clonal hematopoiesis have used conventional BMT techniques involving high-dose irradiation of recipient mice to favor the engraftment of donor bone marrow cells (3-6). However, this myeloablation technique has several disadvantages, including the replacement of cardiac-resident macrophages by donor bone marrow-derived cells that parallels the replacement of cell populations in the bone marrow and blood (Figure 1A). Because irradiation can influence the processes that control clonal hematopoiesis and its cardiovascular impact by damaging the bone marrow niche and heart, we sought to overcome these limitations with an adoptive BMT technique in which unfractionated bone marrow cells are injected into nonirradiated mice, thereby mimicking unperturbed hematopoiesis (20-22). In our experiments, bone marrow cells from donor mice were delivered intravenously to recipient mice as specified in Methods. To maximize the engraftment and expansion of cells in this setting, we intravenously transferred 1.5 $\times 10^{7}$ unfractionated bone marrow cells to each recipient mouse, split into 3 doses over 3 consecutive days. Different CD45.1 versus CD45.2 immune cell populations were defined in bone marrow, blood, and heart, as shown in Supplemental Figure 1; supplemental material available online with this article; https://doi.org/10.1172/jci.insight.135204DS1. As expected, bone marrow delivery to nonconditioned mice led to low-efficiency engraftment of donor bone marrow cells compared with mice treated with lethal irradiation (Figure 1B). The engraftment of donor cells was $3.5 \% \pm 0.55 \%$ white blood cells (WBCs) at 4 weeks after transfer.

To evaluate clonal dynamics of Tet2-deficient cells, we delivered CD45.2 Tet2-WT, Tet2-heterozygous, or Tet2-null bone marrow cells to non-preconditioned CD45.1 recipient mice (Figure 2A). In contrast to WT cells, CD45.2 bone marrow cells from homozygous Tet2-deficient mice expanded progressively from $6.6 \% \pm 0.29 \% \mathrm{WBCs}$ at 4 weeks after transfer to $42.8 \% \pm 3.1 \% \mathrm{WBCs}$ at 8 months after transfer (Figure 2B). The expansion of heterozygous Tet2-deficient cells was slower, suggesting 
A

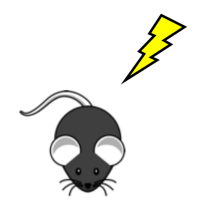

Lethal irradiation

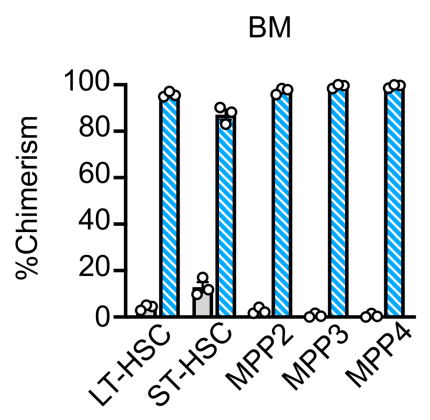

Blood

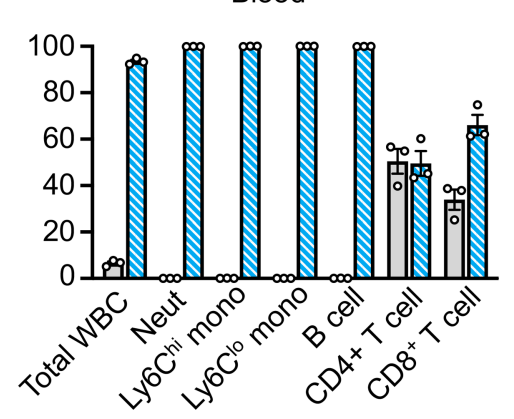

Blood

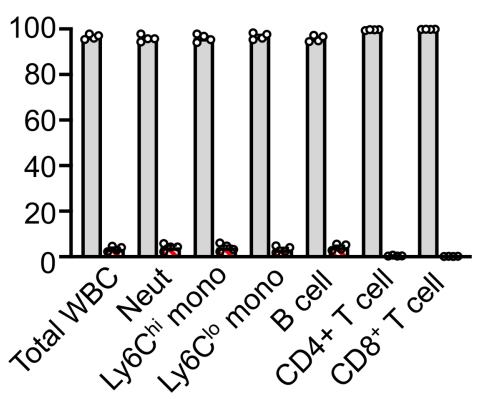

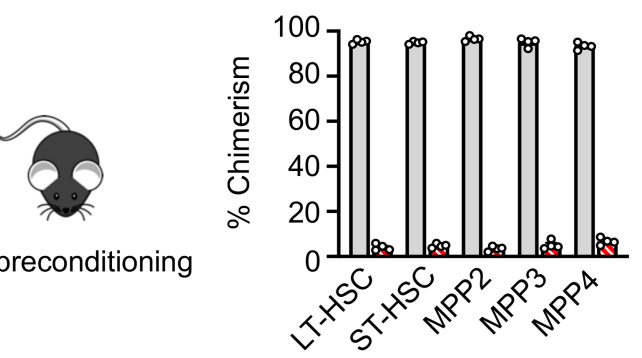

BM

No preconditioning

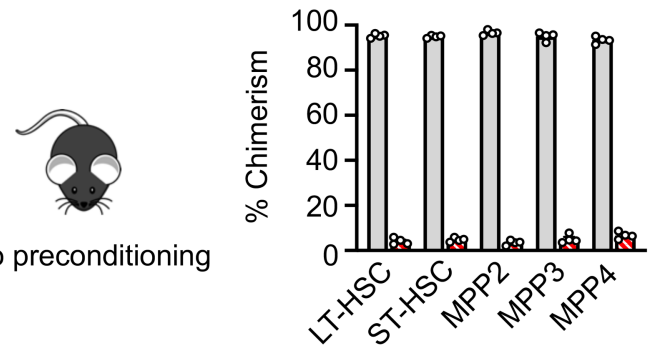

B

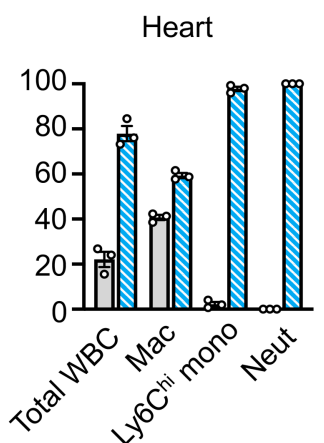

QDonor cells $\square$ Recipient cells

Figure 1. Hematopoietic stem/progenitor cells are engraftable in a nonmyeloablative manner. (A) Flow cytometric analyses of bone marrow, peripheral blood and heart digests 1 month after the transplantation of 5 million bone marrow cells into recipient mice preconditioned with lethal irradiation as specified in Methods $(n=3)$. (B) Flow cytometric analysis of bone marrow, peripheral blood, and heart digests 1 month after the transplantation of $1.5 \times 10^{7}$ unfractionated bone marrow cells over 3 consecutive days to recipient mice without preconditioning as specified in Methods $(n=4)$.

a gene dosage effect. The adoptive transfer of Tet2-deficient cells led to the time-dependent expansion of CD45.2 in all the analyzed blood cell lineages, with a mild myeloid bias (Figure 2B, Supplemental Figure 2A). Expansion into the T cell population occurred to a smaller extent than other blood cell populations $(15.9 \% \pm 1.7 \%$ CD 45.2 Tet2-deficient $\mathrm{T}$ cells at 8 months), presumably because of the slower turnover of these cells in the blood. Although the expansion of Tet2-deficient cells increased the level of chimerism in the various blood cell populations, it did not affect absolute cell numbers. Other hematological parameters, including hemoglobin and platelet counts, did not show significant differences between groups (Supplemental Figure 2B). Collectively, these data indicate that the adoptive transfer of hematopoietic stem and progenitor cells (HSPCs) mimics human clonal hematopoiesis caused by TET2 mutations in the absence of potentially confounding physiological perturbations due to myeloablative transplant conditioning regimens.

Effect of Tet2-deficient HSPC adoptive transfer on the bone marrow compartment. We next assessed the expansion of Tet2-deficient HSPCs in bone marrow cells following the adoptive transfer of WT and heterozygous and homozygous Tet2-deficient cells. Analysis at 8 months after transplant revealed a significant expansion of Tet2-deficient cells in the immature lineage-Sca ${ }^{+}{ }^{+}-\mathrm{Kit}^{+}$(LSK) fractions (Figure 3A). Consistent with this observation, we observed selective expansion of LSK constituent populations, including short-term and long-term (LT-) HSC pools (Figure 3A). Tet2-deficient donor chimerism was also significantly increased in all 3 lineage-primed multipotent progenitor (MPP) populations arising from LT-HSCs $(23,24)$, including myeloid-biased MPP3 and lymphoid-biased MPP4, consistent with expansion of both myeloid and lymphoid cell types in the bone marrow and blood (Figure 3B). Taken together, these data are consistent with prior observations that Tet2-deficient HSCs display a competitive advantage and myeloid bias (25). Along these lines, we found that Tet2-deficient HSCs expressed significantly higher levels of cell cycle genes, including Cdk1, Cdk6, Ccna2, and Foxm1, and lower levels of the cell cycle inhibitor $C d k n 1 a$, relative to either recipient HSCs from the same mice or WT donor HSCs (Figure 3, C and D). Tet2-deficient HSCs also expressed higher levels of $M y c$, which is known to dictate competitive advantage in hematopoietic cells (26) (Figure 3D). 
A

Non-preconditioned mice

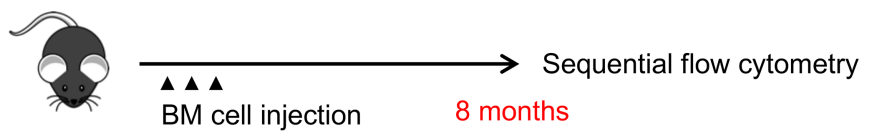

$\left(5 \times 10^{6}\right.$ cells/ injection)

B
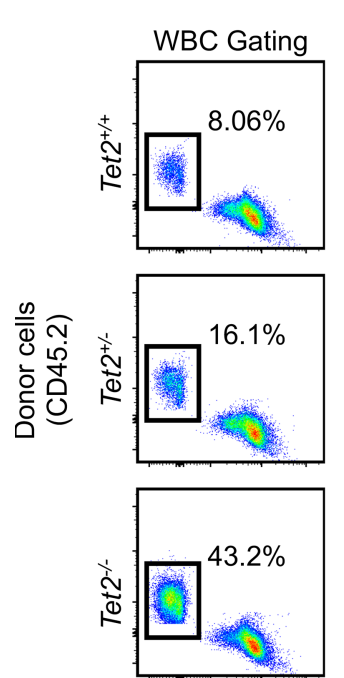

CD45. 1

(Recipient cells)

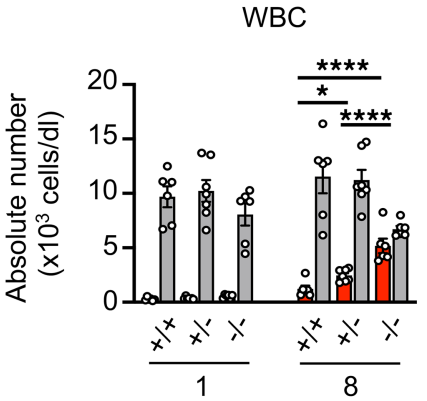

Ly6C ${ }^{\text {lo }}$ monocyte

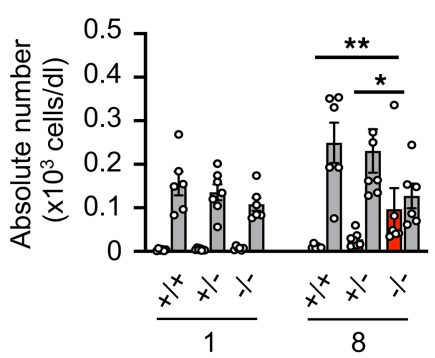

Neutrophil

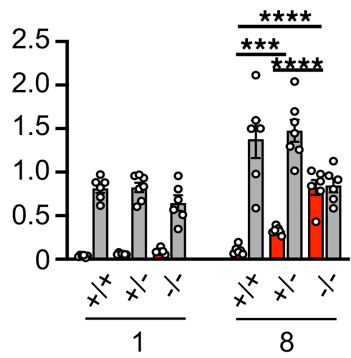

B cell

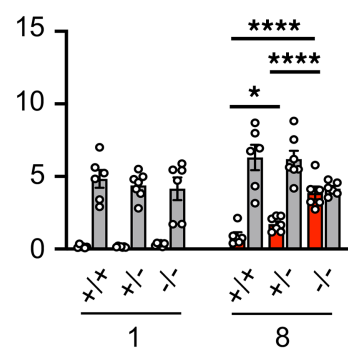

Ly6Chi monocyte

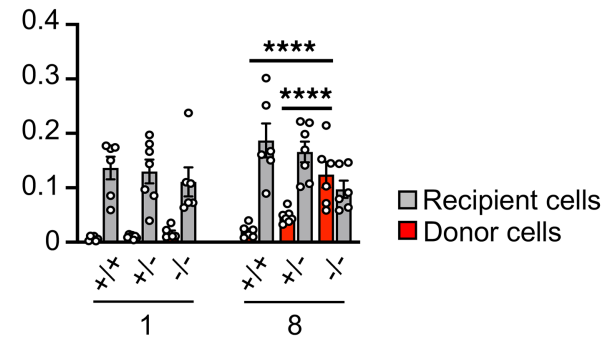

T cell

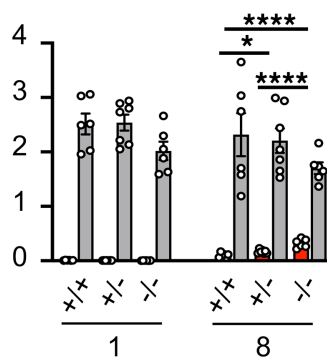

(donor)

(months after BMT)

Figure 2. Transplantation of Tet2 loss-of-function cells in nonconditioned mice leads to a dose-dependent increase in chimerism in peripheral blood. (A) Schematic of this study. A total of $1.5 \times 10^{7}$ unfractionated donor bone marrow cells were sequentially injected into non-preconditioned B6 CD45.1 Pep Boy recipients over 3 consecutive days. Donor cells were obtained from either C57BL/6J WT mice (Tet2 $2^{+/+}$) or Tet2-deficient mice $\left(\mathrm{Tet2}^{+/-}\right.$and $\left.\mathrm{Tet2}^{-/-}\right)$. Absolute number and the chimerism of test cells in peripheral blood were evaluated by sequential flow cytometry analysis. (B) Representative flow data for WBCs and quantitation of flow cytometry analysis of peripheral blood at 1 month and 8 months to show increased chimerism of donor-derived cells in mice transplanted with Tet2-deficient cells ( $n=6-7$ per genotype). Statistical analysis was performed with 2-way repeated-measures ANOVA with Tukey's multiple-comparisons tests. ${ }^{*} P<0.05,{ }^{* *} P<0.01,{ }^{* * *} P<0.001$, and ${ }^{* * * *} P<0.0001$.

To further characterize the competitive advantage of Tet2-deficient HSCs, we sorted Tet2-deficient donor HSCs and recipient HSCs from the same chimeric mice, cultured them in a 1:1 ratio, and monitored expansion of donor and recipient cells over time (Supplemental Figure 3A). Tet2-deficient donor cells exhibited a faster rate of expansion and rapidly overtook the culture (Supplemental Figure 3, B and C). Altogether, these data indicate that Tet2-deficient HSPCs are capable of selectively expanding in the bone marrow, thereby driving the overrepresentation of Tet2-deficient myeloid and lymphoid cells that is observed in the peripheral blood.

Analysis of tissue-resident hematopoietic cell chimerism. Flow cytometric analysis of digested hearts revealed that donor Tet2-deficient HSPCs contributed to cardiac immune populations, including neutrophils, Ly6C $\mathrm{Ch}^{\text {hi }}$ monocytes, macrophages, B cells, and $\mathrm{T}$ cells to a greater degree than that observed in donor WT cells (Figure 4, A and B, and Supplemental Figure 4). This increase in chimerism was gene dose dependent, with the transplantation of homozygous Tet2-deficient cells having a greater impact than the transplantation of heterozygous Tet2-deficient cells at the 8-month time point (for example, $8.8 \% \pm$ $1.8 \%, 23.7 \% \pm 3.5 \%$, and $11.0 \% \pm 0.99 \%$ using donor cells from WT mice and $52.9 \% \pm 3.8 \%, 40.1 \% \pm$ $3.9 \%$, and $24.2 \% \pm 1.6 \%$ using donor cells from homozygous Tet2-deficient mice, in neutrophils, Ly6 $\mathrm{C}^{\mathrm{hi}}$ monocytes, and total macrophages, respectively) (Figure 4B). Notably, the degree of chimerism achieved by the adoptive transfer of Tet2-deficient cells was considerably greater in the cardiac neutrophil and Ly $6 \mathrm{C}^{\text {hi }}$ monocyte populations than in the overall macrophage pool. In contrast to hearts, analysis of kidneys or livers from these mice revealed a much greater degree of Tet2-deficient donor chimerism in the macrophage population ( $46.8 \% \pm 3.3 \%$ in kidney and $56.2 \% \pm 4.1 \%$ in liver at 8 months) that was roughly 
A

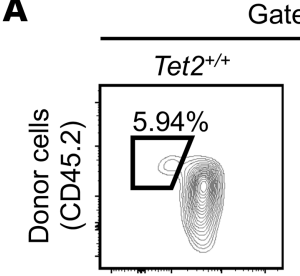
Gated CD48+CD150+LSK

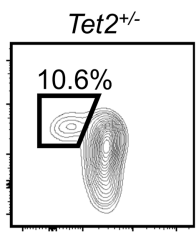

CD45.1

(Recipient cells)

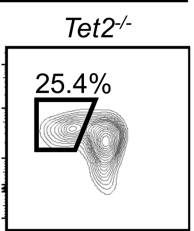

LT-HSC ST-HSC
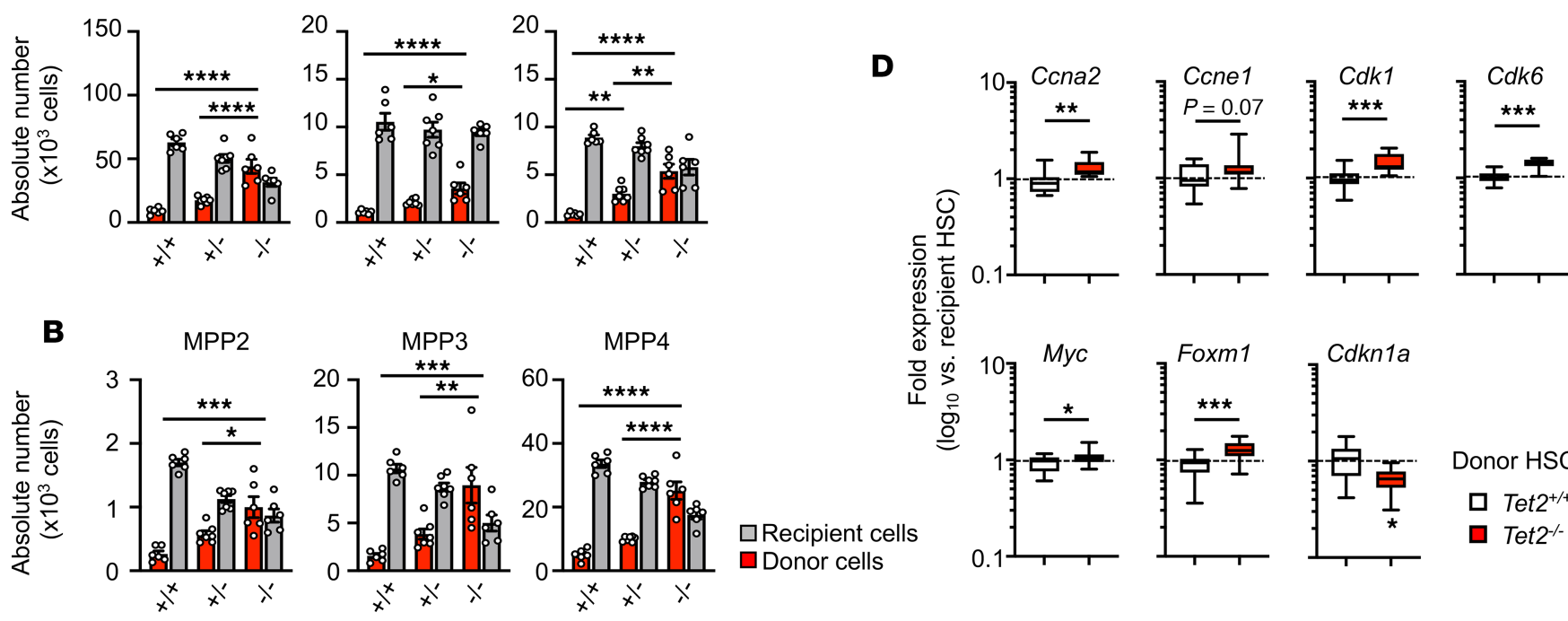

Figure 3. Transferred Tet2-deficient HSCs regulate the transcript signatures of their WT counterparts. Flow cytometry analysis data of bone marrow at 8 months after BMT showing increased chimerism of donor-derived cells in mice transplanted with Tet2-deficient cells ( $n=6-7$ per genotype) in (A) HSCs, with representative long-term (LT-) and short-term (ST-) HSC data shown, and (B) multipotent progenitors (MPPs). Statistical analysis was performed with 1-way ANOVA with Tukey's multiple-comparisons tests. (C) Experimental design and (D) gene expression analysis of cell cycle regulators in Tet2-sufficient and Tet2-deficient donor HSCs isolated from bone marrow ( $n=13-16$ per genotype). The box plots depict the minimum and maximum values (whiskers), the upper and lower quartiles, and the median. The length of the box represents the interquartile range. Data are expressed as log fold change relative to recipient HSCs. Statistical analysis was performed with Mann-Whitney $U$ test. ${ }^{*} P<0.05,{ }^{* *} P<0.01,{ }^{* *} P<0.001$, and ${ }^{* * * *} P<0.0001$.

equivalent to the degree of chimerism achieved in the monocyte and neutrophil populations of these tissues (Supplemental Figure 5, A and B). Collectively, these data indicate tissue specificity in the dynamics of macrophage replacement by Tet2-deficient cells. Whereas kidney and liver macrophages can be rapidly replaced by monocyte-derived macrophages, the macrophages in the noninjured heart appear to be more long-lived and relatively impervious to replacement by Tet2-deficient bone marrow cells.

We further analyzed subpopulations of cardiac macrophages based on the surface expression of CCR2 and major histocompatibility complex II (MHCII). By 8 months after adoptive transfer, WT bone marrow donor cells contributed to all 4 subpopulations with different dynamics (Figure 4C). The Tet2-deficient donor cells contributed a high degree of chimerism to the resident CCR2 ${ }^{+} \mathrm{MHCII}{ }^{\text {hi }}$ macrophage population that is derived from circulating monocytes and exhibits a relatively rapid turnover rate (27). However, there was minimal expansion of Tet2-deficient cells into the CCR2 ${ }^{+} \mathrm{MHCII}^{\mathrm{lo}}, \mathrm{CCR}^{-} \mathrm{MHCII}^{\mathrm{hi}}$, and CCR2-MH$\mathrm{CII}^{\mathrm{Io}}$ populations of cardiac immune cells, which represent the yolk sac-derived macrophage populations. Furthermore, the adoptive transfer of Tet2-deficient HSPCs had no detectable impact on the absolute number of cells of any cardiac immune cell population that was analyzed (Figure 4, B-D). These data are consistent with a model in which the competitive advantage of Tet2-deficient cells in the bone marrow leads to the differential colonization of cardiac tissue with Tet2-deficient myeloid cells without affecting the overall distributions of these cells in the cardiac tissue.

Transcriptomic analysis of cardiac macrophages derived from donor HSPCS. To assess in an unbiased fashion the transcript expression profiles of the donor bone marrow-derived WT and Tet2-deficient macrophages that had infiltrated the heart, we performed ultralow-input RNA-Seq analysis. As shown in Figure 5A, 1363 
A

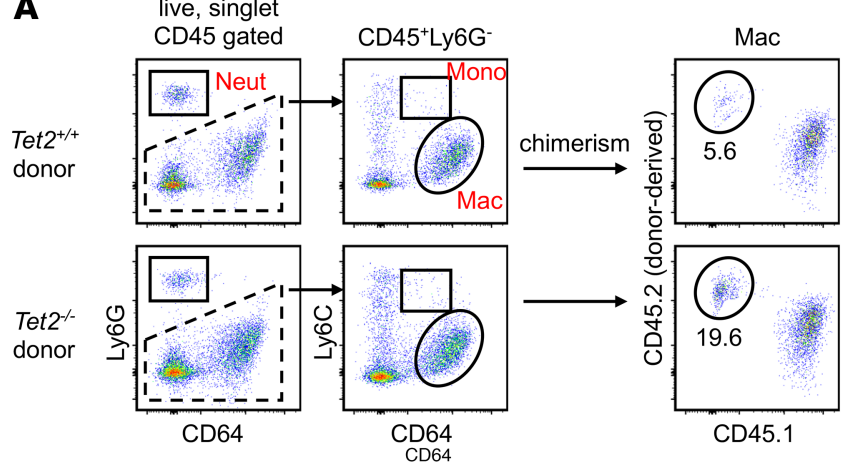

C

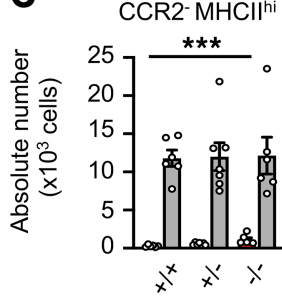

$\mathrm{CCR} 2^{+} \mathrm{MHCll}^{\mathrm{hi}}$

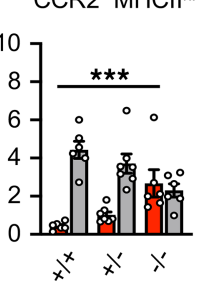

B

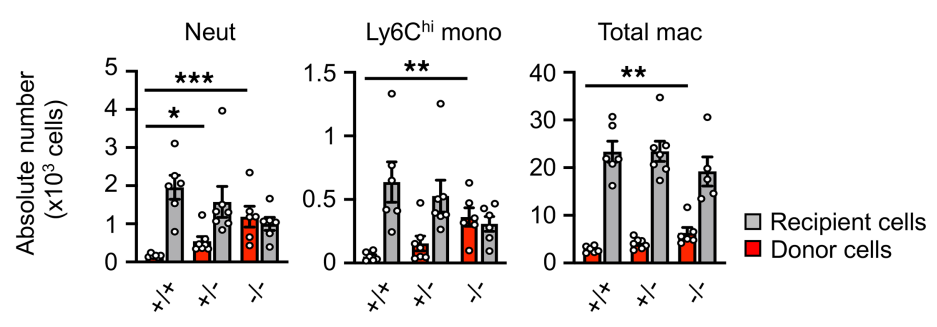

Figure 4. Tet2-mediated clonal hematopoiesis modulates age-related immunological remodeling of the heart. (A) Representative flow cytometry data of heart digests at 8 months after BMT. Cells were defined as neutrophils (Neut; CD45+Ly6C+), Ly6C ${ }^{\text {hi }}$ monocytes (Ly6C ${ }^{\text {hi }}$ mono; CD45+Ly6C-CD64+Ly6C hi), or macrophages (Mac; $C D 45^{+}$Ly6C $-C^{-} D 644^{+}$Ly6 $C^{\circ}$ ). (B) Flow cytometry analysis of immune cells in the heart shows increased chimerism of donor-derived cardiac immune cells in mice transplanted with Tet2-deficient cells ( $n=6-7$ per genotype). Statistical analysis was performed with Kruskal-Wallis $H$ test with Dunn's multiple-comparisons tests (Neut, Total mac) and 1-way ANOVA with Tukey's multiple-comparisons test (Ly6Chi mono). (C) Flow cytometry analysis of subpopulations of cardiac macrophages shows the higher replacement of resident macrophage in mice transplanted with Tet2-deficient cells ( $n=6-7$ per genotype). Statistical analysis was performed with Kruskal-Wallis $H$ test with Dunn's multiple-comparisons tests (Neut, Total mac) and 1 -way ANOVA with Tukey's multiple-comparisons test (Ly6C ${ }^{\text {hi }}$ mono). (D) Flow cytometry analysis of cardiac macrophages showing the fractions of total CD45+ macrophage subpopulations ( $n=6$ per genotype). ${ }^{*} P<0.05$, ${ }^{* *} P<0.01$, and ${ }^{* * *} P<0.001$.

CD45.2 $2^{+} \mathrm{CD}^{2} 4^{+} \mathrm{Ly} 6 \mathrm{C}^{-}{ }^{-} \mathrm{Ly} 6 \mathrm{G}^{-}$cells were isolated by FACS from hearts obtained from CD45.1 recipient mice 8 months after undergoing nonmyeloablative engraftment with either WT or Tet2-deficient bone marrow cells. Principal component analysis revealed that Tet2-deficient cardiac macrophages were distinct from the WT macrophages (Figure 5B). Using a statistical cutoff of $P<0.05$ transcripts per million value of at least 4 and 1.5-fold or more differential expression, we identified 1972 differentially expressed genes between WT and Tet2-deficient macrophages (Figure 5C). Among these, 444 genes were upregulated in the Tet2-deficient condition, but the majority (1528) of these differentially regulated genes were downregulated. A heatmap representation of genes that were highly differentially expressed, in the most overrepresented pathway analyses, for the 3 replication sets of WT and Tet2-deficient conditions is shown in Figure 5D. Using the WebGestalt tool, we analyzed enriched biological processes in the 444 upregulated genes. This analysis revealed an overrepresentation of transcripts in the immune response and regulation of immune system process categories (Table 1, Supplemental Tables 1 and 3). These gene sets included cytokines (Ccl17, Il1b, Illrn), cytokine receptors (Cxcr3, Ccrl2, Illr2), and interferon-related genes (OasIg, Oas2, Oas3, Ifi206, Ifi209, Irf7). The analysis of specific cytokines previously examined in a left anterior descending artery ligation model of Tet2-mediated clonal hematopoiesis revealed the upregulation of $I l 1 b, I l 6, T n f, C c l 2$, and $C c l 5$ by 1.9-fold $(P=0.001)$, 1.5 -fold $(P=0.06), 1.3$-fold $(P=0.10), 1.2$-fold $(P=0.24)$, and 2.3 -fold $(P=0.08)$, respectively. Overall, the characterization of transcripts upregulated in the infiltrating Tet $2^{-1-}$ macrophages supports the concept that Tet2 deficiency upregulates inflammatory pathways that have previously been implicated in clonal hematopoiesis-accelerated cardiac pathology (4-6).

In contrast to genes that were upregulated by Tet 2 deficiency in macrophages, downregulated transcripts displayed enrichment in the categories of regulation of cell differentiation and neurogenesis, among others (Table 1, Supplemental Tables 2 and 3). Based on these findings we analyzed donor-derived Lyve1-MH$\mathrm{CII}^{\mathrm{hi}}$ and $\mathrm{Lyve}^{+} \mathrm{MHCII}^{\mathrm{Io}}$ cardiac subpopulations of interstitial macrophages that are cardioprotective and localized adjacent to nerve bundles and fibers and to blood vessels, respectively (28). To compensate for the elevation in the donor chimerism of peripheral blood Ly6 $\mathrm{C}^{\mathrm{hi}}$ monocytes, the percentage of CD45.2 signal in the cardiac macrophage subpopulations was normalized to the percentage of CD45.2 signal in the 
A

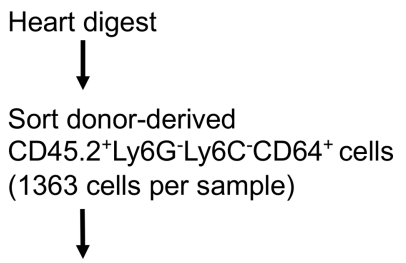

RNA sequencing
B

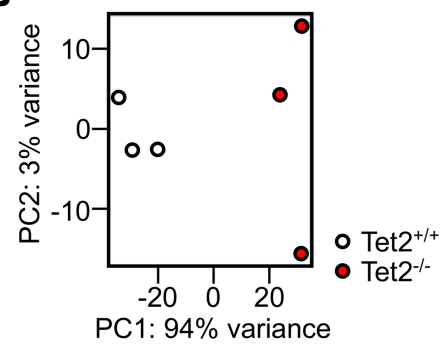

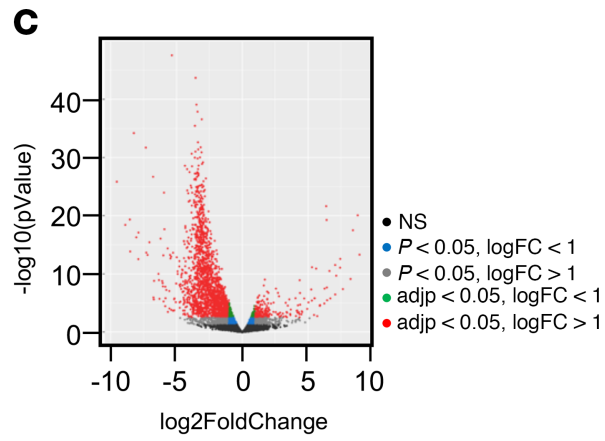

D

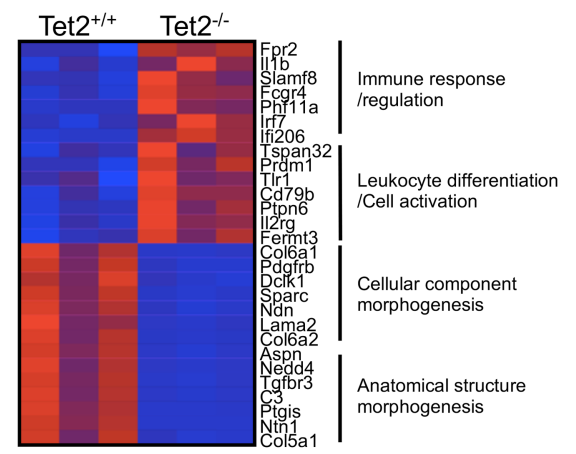

$\mathbf{E}$

$$
\text { min max }
$$

Figure 5. RNA-Seq analysis of Tet2-sufficient and Tet2-deficient donor-derived cardiac macrophages. (A) Scheme of the study. Donor bone marrowderived macrophages were defined as the CD45.2+CD64+Ly6C-Ly6C- population. At 8 months after adoptive transfer, 1363 cells were sorted from mice and used for ultralow-input RNA-Seq. (B) Principal component analysis of ultralow-input RNA-Seq data obtained from sorted donor bone marrow-derived cardiac macrophages of Tet2-sufficient and Tet2-deficient mice ( $n=3$ per genotype). (C) Volcano plots showing the number of genes differentially expressed between cardiac macrophages derived from WT versus Tet2-deficient bone marrow cells. (D) Heatmap of a select group of the most highly differentially expressed genes from the highest ranked annotation categories comparing cardiac macrophages derived from WT versus Tet2-deficient bone marrow cells. (E) Result of donor chimerism of cardiac subpopulations normalized by donor chimerism of peripheral blood Ly6Chi monocytes ( $n=6$ per genotype). Statistical analysis was performed with 2-tailed unpaired Student's $t$ test. ${ }^{*} P<0.05$, and ${ }^{* * *} P<0.0001$.

peripheral blood $\mathrm{Ly}^{6} \mathrm{C}^{\text {hi }}$ monocyte population. Whereas the contribution of CD45.2 donor cells to the chimerism of $\mathrm{CCR} 2^{+} \mathrm{MHCII}{ }^{\mathrm{hi}}$ macrophages was comparable between Tet2-deficient and WT cells, Tet2-deficient cells contributed less efficiently to the chimerism of $\mathrm{Lyve} 1^{-} \mathrm{MHCII}{ }^{\mathrm{hi}}$ and $\mathrm{Lyve} 1^{+} \mathrm{MHCII}^{\mathrm{lo}}$ macrophage subpopulations (Figure 5E). Collectively, these data show that Tet2-deficient donor cells display altered phenotypic properties and skewing in their differentiation to various cardiac macrophage subpopulations.

Accelerated age-dependent cardiac dysfunction in mice receiving Tet2-deficient bone marrow cells. Experimental studies indicate that clonal hematopoiesis can promote cardiac dysfunction in models of injury (4-6). Thus, it was of interest to assess whether cardiac dysfunction could be detected in this adoptive transfer model of Tet2-mediated clonal hematopoiesis in the physiological context of aging in the absence of injury. As mice age, they develop cardiac dysfunction that is associated with greater inflammation (29), and as expected, greater neutrophil, monocyte, and macrophage infiltration was observed in 20-month-old WT mice compared with 8-week-old mice (Supplemental Figure 6).

Serial echocardiographic measurements revealed that cardiac dysfunction could be detected approximately 8 months after the adoptive transfer of homozygous Tet2-deficient bone marrow (Supplemental Figure 7A). At this time point, mice transplanted with Tet2-deficient bone marrow cells also showed significantly higher serum brain natriuretic peptide (BNP) levels (Supplemental Figure 7B), a marker of heart failure. In a separate control experiment, no differences could be observed between sham mice (no BMT) and mice transplanted with WT bone marrow (Supplemental Figure 7C). Finally, an analysis of histological sections for TUNEL ${ }^{+}$cells indicated a low level of apoptosis that did not differ between the adoptive transfer of $\mathrm{Tet}^{+/+}$and of $\mathrm{Tet} 2^{-/-}$bone marrow (data not shown).

In a separate group of mice followed for 18 months, the adoptive transfer of Tet2-deficient bone mar- 
Table 1. Overrepresentation analysis showing pathways and specific genes enriched in Tet2-sufficient and Tet2-deficient donor bone marrow-derived cardiac macrophages

\begin{tabular}{cc}
\hline $\begin{array}{c}\text { Enriched in Tet2-sufficient cardiac macrophages } \\
\text { Regulation of cell differentiation }\end{array}$ & $\begin{array}{c}\text { Enriched in Tet2-deficient cardiac macrophages } \\
\text { Neurogenesis }\end{array}$ \\
\hline Cell projection organization & Regulation of immune system process \\
\hline Response of endogenous stimulus & Cell activation \\
Regulation of anatomical structure morphogenesis & Response to cytokine \\
Cellular component morphogenesis & Immune effector process \\
\hline
\end{tabular}

row cells led to a high degree of donor cell chimerism in the neutrophils, Ly6 $\mathrm{C}^{\text {hi }}$ monocytes, and total macrophages in the hearts of the homozygous Tet2-deficient condition (Figure 6, A and B). Serial, noninvasive echocardiography revealed a progressive decline in cardiac ejection fraction (EF) that was associated with an increase in posterior wall dimension (PWd) and left ventricle end-systolic volume (LVESV) in mice that received the Tet2-deficient donor cells (Figure 6C). Consistent with the echocardiography results, heart weight and myocyte hypertrophy were increased in mice transplanted with Tet2-deficient cells, but mice did not display a detectable difference in blood pressure as measured by tail cuff plethysmography (Figure 6, D-G). Picrosirius red and fast green staining showed that the hearts from mice receiving the Tet2-deficient cells were more fibrotic (Figure $6 \mathrm{H}$ ). Collectively, these data document that the expansion of Tet2-deficient hematopoietic cells in aging, nonconditioned mice is sufficient to induce cardiac dysfunction in the absence of external injurious stimuli.

\section{Discussion}

Our previous studies identified the mechanistic linkage between clonal hematopoiesis and cardiovascular disease using a competitive BMT technique where a high dose of radiation is used to deplete the hematopoietic system of recipient mice to ensure high levels of donor cell engraftment (3-6). Although the transplantation of HSCs into myeloablated mice is widely used to delineate the properties of hematopoietic cell differentiation, high-dose radiation has several disadvantages, which limits its usefulness as a method to model human clonal hematopoiesis. It is recognized that unperturbed, native hematopoiesis and postmyeloablation hematopoiesis represent significantly different physiologies of blood development $(30,31)$. In addition, radiation conditioning directly damages cardiovascular tissues (18) and, as shown here, leads to significantly accelerated replacement of cardiac-resident macrophages by donor bone marrow-derived cells that parallels the rapid replacement of cell populations in the bone marrow. Emerging evidence shows that resident cardiac macrophages exhibit broad diversity with distinct properties from the circulating monocyte-derived macrophages and that some fractions of resident cardiac macrophages exert regenerative and protective functions in models of injury ( 32 , 33). Thus, it would be ideal to maintain the endogenous immune regulatory properties of the heart in studies of clonal hematopoiesis-mediated cardiovascular disease. In this regard, the rapid replacement of bone marrow and cardiac-resident cells in radiation-conditioned models is unlikely to recapitulate the gradual dynamics of resident cardiac macrophage turnover and changes in cardiac immune regulatory properties that influence cardiovascular disease development in the context of human clonal hematopoiesis.

To overcome these limitations, we used an adoptive BMT technique in which unfractionated bone marrow cells are injected into unconditioned mice (20-22). In our experiments, the delivery of WT CD45.2 bone marrow cells to mice expressing CD45.1 mice led to a low but stable engraftment of donor-derived LT-HSCs and various HSPC fractions, as well as donor contribution to circulating leukocyte populations and resident immune cell populations in the heart and other tissues. Strikingly, the adoptive transfer of Tet2-deficient bone marrow cells led to a gradual expansion of donor cells into all these cell populations relative to WT donor bone marrow following adoptive transfer, with homozygous Tet2-deficient cells achieving a higher degree of chimerism than heterozygous Tet2-deficient cells following implantation. Collectively, these data show that Tet2-deficient HSPCs are capable of preferential expansion in unperturbed bone marrow.

Consistent with the notion that Tet 2 deficiency confers a competitive advantage to HSPCs, donor-derived Tet2-deficient LT-HSCs expressed higher levels of genes associated with proliferation and increased cell fitness, such as $M y c$, and exhibited a growth advantage in a competitive cell culture assay. The capacity of Tet2- 
A

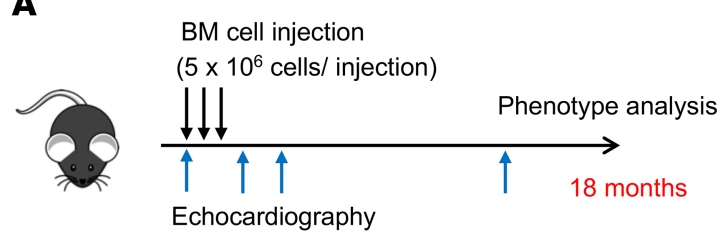

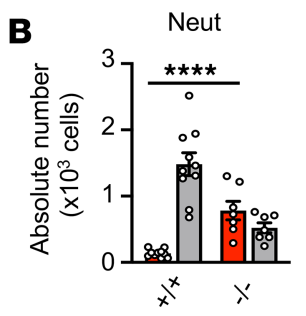

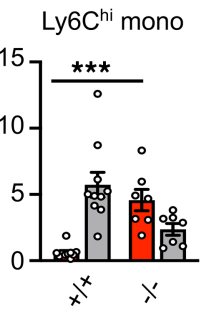

Total mac

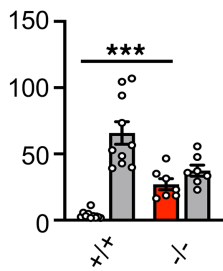

$\square$ Recipient cells $\square$ Donor cells
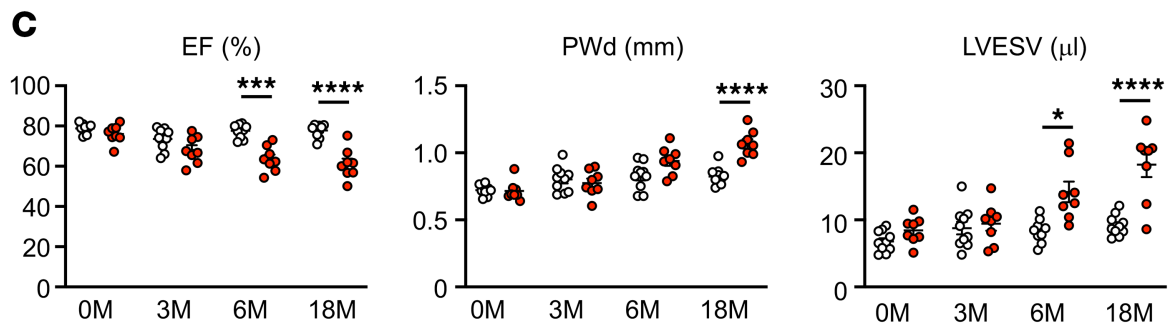

$\operatorname{LVEDV}(\mu \mathrm{l})$
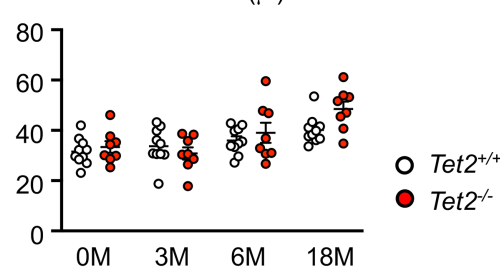

D

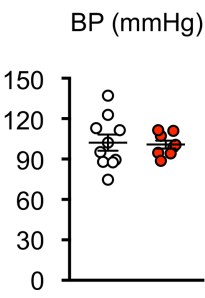

E

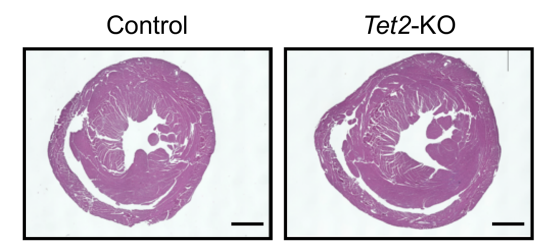

$\mathbf{F}$

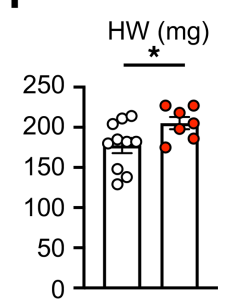

G

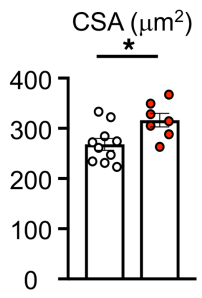

H

Fibrosis (\%)

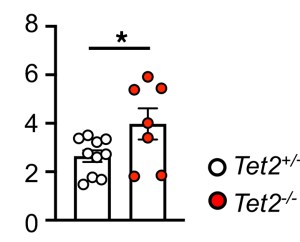

Figure 6. Tet2-mediated clonal hematopoiesis accelerates age-related cardiomyopathy. (A) Schematic of this study. A total of $1.5 \times 10^{7}$ unfractionated donor bone marrow cells were sequentially injected into non-preconditioned B6 CD45.1 Pep Boy recipients. Donor cells were obtained from either C57BL/6) WT mice $\left(\right.$ Tet2 $\left.^{+/+}\right)$or Tet2-deficient mice $\left(\right.$Tet2 $\left.{ }^{-/}\right)$. Echocardiography was performed at the indicated time points. Mice were euthanized for the analysis 18 months after BMT. (B) Flow cytometry analysis of immune cells in the heart shows increased chimerism of donor-derived cardiac immune cells in mice transplanted with Tet2-deficient cells ( $n=7-10$ per genotype). Statistical analysis was performed with 2-tailed unpaired Student's $t$ test (Neut) and Mann-Whitney $U$ tests (Ly6C ${ }^{\text {hi }}$ mono, Total mac). (C) Data of sequential echocardiography analysis to show progressive decrease in cardiac function in mice with Tet2-mediated clonal hematopoiesis ( $n=7-10$ per genotype). Statistical analysis was performed with 2-way repeated-measures ANOVA with HolmŠídák multiple-comparisons tests. (D) Tail cuff-measured blood pressure of each group at study end to show no obvious difference in each group ( $n=7-10$ per genotype). Statistical analysis was performed with 2-tailed unpaired Student's $t$ test. (E) Representative data of histological analysis of the heart from mice transplanted with Tet2-sufficient and -deficient bone marrow cells at the end of study. Scale bar: $600 \mu \mathrm{m}$. (F) Heart weight of the mice from each group ( $n=7-10$ per genotype). Statistical analysis was performed with 2-tailed unpaired Student's $t$ test. (C) Cross-sectional area (CSA) in left ventricles 18 months after BMT was assessed by wheat germ agglutinin staining from heart of Tet2 ${ }^{+/+}$BMT $(n=10)$ mice and Tet2 $2^{-/-}$BMT mice $(n=7)$. Staining shows that the heart displays greater hypertrophy of the cardiac myocytes in Tet2 ${ }^{-1-}$ BMT mice. Statistical analysis was performed with 2-tailed unpaired Student's $t$ test. (H) Quantitative analysis of cardiac sections stained with Picrosirius red showing that mice with Tet2-mediated clonal hematopoiesis exhibit enhanced fibrosis ( $n=7-10$ per genotype). Statistical analysis was performed with 2-tailed unpaired Student's $t$ test. ${ }^{*} P<0.05,{ }^{* *} P<0.001$, and ${ }^{* * *} P<0.0001$.

deficient HSPCs to undergo preferential expansion in the bone marrow could be a consequence of overactive responses relative to WT HSPCs to tonic levels of inflammatory cytokines, such as IL-1 $\beta / \mathrm{IL}-6$ (3-5), because Tet2-deficient HSPCs display enhanced responses to inflammation and are protected from inflammation-induced damage (34). Thus, the nonconditioned adoptive transfer model described here can be leveraged in future studies to identify the extent to and mechanism(s) by which interplay between Tet2-deficient HSPCs and the bone marrow microenvironment drives clonal hematopoiesis, particularly in contexts where clonal hematopoiesis is prevalent, such as aging and exposure to genotoxic stress.

There is a growing appreciation that the heart is an immunologically active tissue under homeostatic conditions and that its resident immune cells play important roles in cardiac maintenance and repair (32). However, these populations are severely disrupted following irradiation, limiting the utility of radiation conditioning-based models for studying the link between the dynamics of mutant HSPCs in the bone marrow and their infiltration into the heart during clonal hematopoiesis. The nonirradiated heart contains populations of tissue-resident macrophages that are derived from progenitors located in the yolk sac and fetal liver and seeded during organogenesis $(27,35)$ These embryo-derived macrophages are maintained by their local proliferation under homeostatic conditions in mice and humans, but injury to the heart will result in the rapid replacement 
of tissue-resident macrophages with bone marrow-derived macrophages $(27,32,33,36)$. It is also reported that blood monocyte-derived macrophages gradually replace embryo-derived cardiac-resident macrophages with aging to extents that differ between the different macrophage subpopulations (37). In this regard, CCR2 ${ }^{+} \mathrm{MH}_{-}^{-}$ $\mathrm{CII}^{\text {hi }}$ macrophages are predominantly derived from circulating monocytes produced by bone marrow HSPCs, whereas CCR2- ${ }^{-} \mathrm{MHCII}^{\mathrm{hi}}$ and $\mathrm{CCR} 2^{-} \mathrm{MHCI}^{\mathrm{lo}}$ macrophages are functionally distinct populations of embryonic origin $(27,33)$. The bone marrow-derived $\mathrm{CCR} 2^{+} \mathrm{MHCII}{ }^{\text {hi }}$ population expresses high levels of cytokines and chemokines that can contribute to cardiac damage. On the other hand, the embryo-derived CCR2-MHCII ${ }^{\mathrm{hi}}$ and CCR2- $\mathrm{MHCII}^{\text {lo }}$ macrophage populations express low levels of inflammatory mediators and high levels of matrix proteins and growth factors that participate in cardiac protection and regeneration. Here, we find that adoptively transferred Tet2-deficient donor bone marrow cells display differential expansion kinetics into various cardiac immune cell populations. Whereas the degree of Tet2-deficient cell chimerism in cardiac neutrophils mirrors that of the chimerism of neutrophils in the blood, the expansion of Tet2-deficient cells into the total cardiac macrophage population is relatively attenuated. These findings are in contrast to the higher levels of chimerism that are observed in the macrophage compartments of the kidney and liver, presumably because these tissues display higher levels of macrophage turnover by monocyte-derived macrophages. Our analysis of macrophage subpopulations in the heart revealed that Tet2-deficient CD45.2 cells preferentially expand into the $\mathrm{CCR} 2^{+} \mathrm{MHCII}{ }^{\mathrm{hi}}$ subgroup, which undergoes rapid turnover and is replenished by bone marrow-derived monocytes. In contrast, there was little or no expansion of Tet2-deficient cells into the cardiac-resident CCR2-MH$\mathrm{CII}^{\text {hi }}$ and CCR2 ${ }^{-} \mathrm{MHCII}^{\mathrm{lo}}$ fractions, consistent with their embryo-derived ontogeny. Furthermore, though the fraction of Tet2-deficient myeloid cells in the heart increased over time, the expansion of Tet2-deficient cells did not detectably affect the absolute numbers of immune cells within the heart, including the embryo-derived macrophage populations. Such findings are consistent with the dynamics of Tet2-deficient HSPCs and myeloid cell expansion in the bone marrow of the adoptively transferred mice. Hence, our model provides a potentially more faithful recapitulation of the dynamics and origin of cardiac-resident myeloid cells, which is crucial for understanding their participation in cardiovascular disease in the context of clonal hematopoiesis.

To understand how Tet2 deficiency in hematopoietic cells could promote cardiac pathology, we performed an unbiased transcriptomic analysis of donor-derived cardiac macrophages isolated from the hearts of mice that received an adoptive transfer of bone marrow from WT or Tet2-deficient mice. Surprisingly, only 444 of the $1972(\sim 22 \%)$ differentially expressed genes were upregulated, suggesting that Tet 2 inactivation predominantly leads to downregulation of gene expression. Tet 2 is a multifunctional epigenetic regulator that is capable of activating or silencing genes through distinct mechanisms. Tet2 is most widely appreciated for its gene activating function through its ability to oxidize 5-methylcytosine in DNA (38, 39). However, Tet2 can also inhibit gene expression via recruitment of histone deacetylases (HDACs) to target genes (3). Thus, Tet2 inactivation can promote aberrant gene expression because of compound loss of HDAC and DNA demethylation activity. In this regard, the proinflammatory actions of Tet2 deficiency have been linked to loss of the HDAC suppressive function, and this is consistent with the gene sets overrepresented in the Tet2-deficient bone marrow-derived macrophages isolated from the heart, including $I l 1 b$, Ccl17, and Ccrl2. Accordingly, we have previously shown that Tet2 deficiency leads to an upregulation of IL-1 $\beta$ /IL-6 signaling in macrophages $(3,5)$, and evidence suggests that these cytokines causally contribute to cardiovascular pathologies $(9,40)$. Hence, our data support a model in which expansion of Tet2-deficient HSPCs in bone marrow leads to overproduction of progeny immune cells with aberrant proinflammatory properties, which subsequently populate the cardiac tissue and promote cardiovascular disease.

The analysis of genes downregulated by the loss of Tet 2 revealed that gene categories of regulation of cell differentiation and neurogenesis, among others, were predominantly overrepresented. These findings appear to align with the finding that Tet2-deficient progenitor cells are impaired in their ability to differentiate into the interstitial subpopulations of macrophages that are reported to promote the functionality of the cardiac neuronal and vascular cells. These individual macrophage subpopulations associate with neurons and blood vessels within the tissue, express distinct transcriptional profiles that reflect their microanatomic locations, and function to support neuronal and vascular beds $(28,41)$. In the heart, a Lyve $1^{-} \mathrm{MHCI}{ }^{\mathrm{hi}}$ macrophage population is localized adjacent to nerve bundles and fibers, and Lyve $1^{+} \mathrm{MHCII}{ }^{\mathrm{lo}}$ macrophages are localized adjacent to blood vessels; both of these populations are reported to arise from bone marrow-derived Ly6 $\mathrm{C}^{\text {hi }}$ monocytes (28). Employing the adoptive bone marrow transfer system, we find that the Tet2-deficient bone marrow HSPCs contribute less efficiently to the chimerism of Lyve ${ }^{-} \mathrm{MHCII}^{\mathrm{hi}}$ and $\mathrm{Lyve} 1^{+} \mathrm{MHCII}{ }^{\mathrm{lo}}$ macrophage subpopulations in the myocardium. Because these niche-specific macrophage subpopulations are increasingly 
replaced from the monocyte pool with age (28), it can be speculated that the expansion of Tet2-deficient monocytes under these conditions could ultimately lead to the age-dependent decline in the Lyve $1^{-} \mathrm{MHCII}{ }^{\mathrm{hi}}$ and $\mathrm{Lyve} 1^{+} \mathrm{MHCII}{ }^{\mathrm{lo}}$ cell populations of the myocardium and thereby contribute to the development of age-related cardiomyopathies via neuronal loss, vascular leakage, inflammation, and fibrosis $(28,41)$.

Using the adoptive transfer model, we provide evidence that Tet2-mediated clonal hematopoiesis is sufficient to accelerate age-related cardiomyopathy. Although age is the predominant factor that contributes to the development of clonal hematopoiesis, these data raise the possibility that clonal hematopoiesis can, in turn, affect the processes that promote biological aging. A recent study associated clonal hematopoiesis with age acceleration through the examination of the "epigenetic clock" that assesses the methylation patterns at specific $\mathrm{CpG}$ sites (42). In longitudinal analyses of 2 cohorts, clonal hematopoiesis was found to be associated with 3.7 and 4.5 years of accelerated age, and individuals with TET2-mediated clonal hematopoiesis displayed the greatest accelerations of epigenetic aging (6.1 and 6.4 years) compared with those individuals with no detectable clonal hematopoiesis. The data presented herein raise the possibility that clonal hematopoiesis may have causal roles in promoting biological aging and diminishing health span. Specifically, we find that this model of Tet2-mediated clonal hematopoiesis is sufficient to promote age-related cardiac dysfunction in the absence of injurious stimuli. Age-dependent cardiac myopathy is typically observed by 18 months in mice and characterized by systolic dysfunction, left ventricle hypertrophy, and fibrosis (29). In mice receiving Tet2-deficient HSPCs, contractile dysfunction could be observed as early as 8 months after the adoptive transfer by noninvasive echocardiography. By the 18-month time point, systolic dysfunction, left ventricular hypertrophy, and cardiac fibrosis were observed to a significantly greater extent in the Tet2-deficient condition than in control mice that received an adoptive transfer of WT bone marrow. Although the extent of Tet $^{-1-}$ cell-mediated cardiac dysfunction was less than that observed in surgical models of heart failure, the decline in contractility and the increases in hypertrophy and fibrosis are consistent with the extent of cardiac impairment that is observed in aging humans and animal models $(29,43)$.

We acknowledge that our study has limitations. First, we primarily analyzed different macrophage populations, but other immune cell populations could be contributing to the clonal hematopoiesisinduced cardiac dysfunction. Our rationale for analyzing cardiac macrophages in this adoptive transfer/ aging model is based upon findings that somatic TET2 mutations in the human population display a pronounced myeloid bias (44) and because myeloid-specific ablation of Tet 2 has been shown to be sufficient to confer pathological phenotypes in atherosclerosis and heart failure models $(3,5)$. However, we cannot rule out the effects of Tet2-mediated clonal hematopoiesis on lymphocytes or other populations of bone marrow-derived cells. In this regard, we previously reported that the expansion of Tet2-deficient hematopoietic cells leads to small changes in the frequencies of $\mathrm{T}$ cell subsets in the aorta and aorta-draining mediastinal lymph nodes in a model of atherosclerosis (3). Thus, additional studies are warranted to understand the immune cell types that mediate the pathological actions of TET2-mediated clonal hematopoiesis in the cardiovascular system. Furthermore, we cannot completely rule out that the detrimental effects of clonal hematopoiesis are due at least in part to the reduction in WT immune cell diversity that is a consequence of the expansion of the mutant clone. However, we believe that this scenario is unlikely for a number of reasons. For example, multiple studies indicate that the mutant HSPCs give rise to progeny blood cells with altered immune properties (e.g., overactivation of IL-1 $\beta /$ IL-6 signaling) that appear to be causative in the disease process $(3,5,9,40)$. In addition, experimental studies have shown that pathological phenotypes can be conferred by low (5\%-10\%) variant allele fractions (VAFs) (5) and that VAF levels of $1 \%-2 \%$ are associated with adverse outcomes in patients with heart failure (15). Thus, it is difficult to reconcile how such small losses in immune cell diversity can account for the robust effects of clonal hematopoiesis on cardiovascular pathologies. Finally, we acknowledge that CD45.1 and CD45.2 cells can display modest differences in their expansion kinetics in competitive BMT assays (45); however, we have shown that Tet2-deficient bone marrow cells markedly outcompete WT cells and contribute to cardiac pathology even when they are transplanted into mice with identical genetic backgrounds (4).

Overall, it is tempting to speculate that the development of somatic mutations in the immune system, which become amplified via the clonal expansion of these cells, could have inordinate actions on the aging organism. It is well recognized that the cells of the immune system are nearly ubiquitously distributed throughout the body and they function in tissue surveillance and in the disposal of dead cells, toxins, and pathogens. Thus, the corruption of these cells through clonal hematopoiesis could lead to "inflammaging" $(46,47)$, having deleterious consequences on multiple systems that contribute to increases in morbidity and mortality. 


\section{Methods}

\section{Animals}

C57BL/6J background Tet2-deficient mice (Tet2 ${ }^{-/-}$and Tet2 ${ }^{+/-}$), C57BL/6J WT mice, and B6 CD45.1 Pep Boy mice were obtained from The Jackson Laboratory (stock 023359, 000664, and 002014, respectively). Male mice were used for the in vivo experiments. Mice were housed in a specific pathogen-free animal facility and given food and water ad libitum on a 12-hour light/12-hour dark schedule. Three cohorts of mice were used for the adoptive transfer experiments in this study. One cohort was followed for 18 months, and 2 independent cohorts of mice were followed for 8 months following the bone marrow adoptive transfer.

\section{BMT}

Lethal irradiation method. Lethally irradiated 8- to 12-week-old C57BL/6J WT recipients were transplanted with suspensions of bone marrow cells containing 100\% C57BL/6 CD45.1 Pep Boy mice. Bone marrow cells were isolated from femurs and tibias of donor 6- to 8-week-old mice that underwent euthanasia. Recipient mice were irradiated in a pie cage (Braintree Scientific) to limit mobility and ensure an equal dose of irradiation and were exposed to 2 radiation doses of 5.5 Gy 4 hours apart using an X-RAD 320 Biological Irradiator. After the second irradiation, each recipient mouse was injected with $5 \times 10^{6}$ bone marrow cells via the retro-orbital vein plexus. Sterilized caging, food, and water were provided during the first 14 days after transplantation, and water was supplemented with the antibiotic Sulfatrim (Teva Pharmaceuticals USA, NDC 0703-9526-01).

Non-preconditioning method. C57BL/6 CD 45.1 $1^{+}$Pep Boy mice, 8 to 12 weeks old, were transplanted with suspensions of bone marrow cells containing either 100\% C57BL/6J WT mice or $100 \%$ Tet2-deficient mice $\left(\right.$ Tet $2^{-/-}$and Tet $^{+/-}$). Over 3 consecutive days, $5 \times 10^{6}$ unfractionated bone marrow cells were injected into nonirradiated recipients via retro-orbital vein $\left(1.5 \times 10^{7}\right.$ cells in total).

\section{Hematopoietic cell parameter measurements}

Hematopoietic parameters were analyzed using an Element HT5 Veterinary Hematology Analyzer (Heska). Flow cytometry analysis of peripheral leukocytes, bone marrow cells, and cardiac immune cells was performed as reported in our previous studies $(5,48)$ at the time points indicated in the figures. The antibodies used for flow cytometric analysis are listed in Supplemental Table 4.

Peripheral blood. Peripheral blood cells were obtained from the retro-orbital vein and collected into K2EDTA-added BD microtainer blood collection tubes (BD Biosciences). Red blood cells were lysed with eBioscience 1X RBC Lysis Buffer (Thermo Fisher Scientific, 00-4333-57) for 5 minutes on ice. Incubation with antibodies was performed for 20 minutes at room temperature in the dark.

Bone marrow cells. Bone marrow cells were flushed out from bones (femur and tibia). Red blood cells were lysed with 1X RBC Lysis Buffer for 30 seconds at room temperature. Incubation with antibodies was performed for 20 minutes at room temperature in the dark. For counting cell numbers, 123 count eBeads Counting Beads (Thermo Fisher Scientific, 01-1234-42) were used.

Cardiac immune cells. Hearts were flushed with $15 \mathrm{~mL}$ of cold phosphate-buffered saline (PBS) from the apex. Before perfusion of the heart, the right atrium was excised to facilitate the perfusion. Right ventricle and other extra tissue were removed, and left ventricles were minced and digested in collagenase I (450 U/mL), collagenase XI (125 U/mL), hyaluronidase $(450 \mathrm{UI} / \mathrm{mL})$, and DNase I $(60 \mathrm{U} / \mathrm{mL})$ (C0130, C7657, H3506, and D4513, respectively, MilliporeSigma) at $900 \mathrm{rpm}$ at $37^{\circ} \mathrm{C}$ for 30 minutes using a ThermoMixer C (Eppendorf). Hearts were subsequently homogenized through Falcon Cell Strainers (Thermo Fisher Scientific, 352350). Contaminated red blood cells were lysed with 1X RBC Lysis Buffer for several seconds at room temperature with mild shaking. Incubation with antibodies was performed for 20 minutes at room temperature in the dark. Dead cells were excluded from analysis by Zombie Aqua staining (Invitrogen, Thermo Fisher Scientific, L34957). For counting cell numbers, 123 count eBeads Counting Beads were used. Data are expressed as number of cells/100 mg wet weight of the heart. A BD LSRII Flow Cytometer (Boston University) or a Fortessa (University of Virginia) was used for data acquisition. Cells were defined as described in Supplemental Figure 1. Data were analyzed with FlowJo Software (Tree Star, Inc.). 


\section{Gene expression analysis of HSCs}

Pools of 100 donor or recipient HSCs were sorted into wells of a DNase- and RNase-free 96-well PCR reaction plate containing $5 \mu \mathrm{L}$ CellsDirect $2 \mathrm{X}$ reaction buffer (Life Technologies), spun down at $500 \mathrm{~g}$, snap-frozen, and stored at $-80^{\circ} \mathrm{C}$. RNA was subjected to 18 rounds of specific target amplification with a custom-designed 96-target DeltaGene (Fluidigm) primer panel using SuperScript III RNA polymerase (Life Technologies) and subsequently treated with Exonuclease-I (New England Biolabs) to eliminate excess primers. Following preamplification, samples and primers were mixed with SsoFast EvaGreen Supermix with Low ROX (Bio-Rad), and quantitative real-time PCR reactions were carried out on a 96.96 Dynamic Array IFC for Gene Expression (Fluidigm). Data were analyzed using Fluidigm gene expression software, normalized to Gusb, and differential expression was determined using the $\Delta \Delta \mathrm{Ct}$ approach.

\section{RNA-Seq}

For RNA-Seq analysis of the cardiac macrophages (CD45.2 $\left.{ }^{+} \mathrm{Ly} 6 \mathrm{G}^{-} \mathrm{CD} 64^{+} \mathrm{Ly} 6 \mathrm{C}^{-}\right)$, heart digests were prepared as described above, and sorting was performed on Influx Cell Sorter platform with a 100- $\mu$ m nozzle and flow pressure set to 20 psi (BD). A total of 1363 cells were sorted for each population directly into 1 $\mathrm{mL}$ of Trizol (QIAGEN Inc.) containing 1\% $\beta$-mercaptoethanol. Samples were then sent to GENEWIZ and subjected to library construction and sequencing. The HiSeq4000 platform was used for sequencing to generate paired-end data of $150 \mathrm{bp}$. Sequence alignment and quantitation were performed by Kallisto 0.44 .0 (49). A Mus musculus GRCm38 FASTA transcriptome file from Ensembl database was used as an index for alignment. Differential gene expression analysis was performed using different RStudio analysis packages. For the identification of statistically overrepresented (enriched) pathways among the differentially expressed genes identified by RNA-Seq analysis, the WebGestalt online tool with Gene Ontology (GO) databases was used. After submission of the gene list, overrepresentation analysis on biological processes was performed based on GO with the Mus musculus genome. To identify the most significantly enriched gene sets, redundancy reduction was done with "weighted set cover" method (50). Data were deposited into the National Center for Biotechnology Information's Gene Expression Omnibus database under accession number GSE146812.

\section{Physiological measurements}

Sequential echocardiography was performed at the time points indicated in the figures using the Vevo 2100 ultrasound system equipped with an MS550D probe (VisualSonics, Fujifilm). Mice were anesthetized with isoflurane at a concentration of $5 \%$ (induction phase) and $1 \%-1.5 \%$ (maintenance phase). Each animal was placed on a heated table in a supine position with the extremities tied to the table through 4 electrocardiography leads. Chest fur was removed with a chemical hair remover, and ultrasound gel was applied to the thorax surface. Mice were kept under a shallow anesthesia state (semiawake) by monitoring responses to physical stimuli (tail pinch, toe pinch, etc.), and heart rate was maintained at approximately 500-600 bpm. Left ventricular ejection fraction (LVEF) and LV systolic and diastolic volume were measured from long-axis view by tracing end-diastolic and end-systolic endocardium. LV posterior wall thickness diameter was measured from M-mode images obtained by short-axis view visualizing both papillary muscles. Measurements and analyses of all the echocardiographs were performed in a consistent way using Vevo Lab software (VisualSonics, Fujifilm) by 2 experienced investigators who were blinded to the identity of the experimental groups of mice. Blood pressure was determined by tail cuff plethysmography $(51,52)$.

\section{Histological analysis}

Heart tissues were perfused with cold PBS from the apex and fixed in $10 \%$ formalin at $4^{\circ} \mathrm{C}$ overnight. Samples were processed for paraffin embedding. Sections ( $7 \mu \mathrm{m}$ thick) were deparaffinized and rehydrated. $\mathrm{H} \& \mathrm{E}$ staining was performed using a standard method. Sections were incubated in filtered hematoxylin solution (MilliporeSigma, GHS316) for 2 minutes, washed in tap water, and differentiated in 1\% acidified alcohol. After dehydration in 70\% ethanol, sections were incubated in eosin solution (MilliporeSigma, HT110116) for a few seconds, dehydrated in a series of ethanol baths ( $95 \%$ and $100 \%)$, cleared in xylene, and mounted in permanent mounting medium (Vector Laboratories, H-5000). Images were taken by a Keyence BZ-X710 microscope to show the global change of heart size. To determine the cardiomyocyte CSA, heart sections were stained with Alexa Fluor 488-conjugated wheat germ agglutinin (W11261, Life Technologies) for 1 hour at room temperature. An operator who was blinded to genotype quantified cardiomyocyte CSA by computer-assisted morphometric analysis of microscopy images acquired on a 
Keyence BZ-X710 microscope. The average CSA of 80-120 randomly selected round-shaped cardiomyocytes per sample was used for analysis. For Picrosirius red/fast green staining, sections were incubated with freshly prepared staining buffer $(1.2 \% /$ wt saturated picric acid in water, $0.1 \% /$ wt Fast Green FCF, and $0.1 \% /$ wt Direct Red 80 ) for at least 1 hour at room temperature (197378, F7252, and 365548, respectively, from MilliporeSigma). Sections were washed briefly in acidified water $(0.1 \% / \mathrm{v}$ glacial acetic acid in water) and dehydrated. The slides were mounted by coverslip using permanent mounting medium. The images were analyzed using ImageJ software (NIH) for quantification of fibrosis. Myocardial fibrosis size was expressed as a percentage of total LV area $(53,54)$.

\section{ELISA}

Plasma was prepared from blood by centrifugation at $8000 \mathrm{~g}$ for 10 minutes at $4^{\circ} \mathrm{C}$. Plasma levels of $\mathrm{BNP}$ were measured using the mouse BNP-specific ELISA (RayBiotech, EIAM-BNP-1) according to the manufacturer's protocols.

\section{Cell culture assays}

Purified HSCs were cultured at a 1:1 Tet2-deficient donor/recipient ratio (200 cells each for 400 total) in a sterile, flat-bottom, 96-well tissue culture plate (Costar) in StemPro serum-free medium (Life Technologies) containing $200 \mu \mathrm{L} 10 \mathrm{ng} / \mathrm{mL}$ stem cell factor, FMS-related tyrosine kinase 3 ligand, and IL-11 (PeproTech). Cells were counted after 4 and 8 days' culture on a hemocytometer, using trypan blue to exclude dead cells. For FACS analysis, cultured cells were stained for 30 minutes on ice with anti-Sca1 BV421, anti-c-Kit APC/Cy7, anti-Mac-1-PE/Cy7, and anti-Fc $\gamma$ R-APC. Cells were subsequently washed, resuspended in staining medium containing $1 \mu \mathrm{g} / \mathrm{mL}$ propidium iodide for dead cell exclusion, and analyzed on a FACSCelesta (BD).

\section{Statistics}

All the statistical analysis was performed with GraphPad Prism 8 software (GraphPad Software). Shapiro-Wilk normality test was performed to evaluate data distribution. Homogeneity of variance was evaluated by $\mathrm{F}$ test. Normally distributed data with 1 variable were analyzed by 2-tailed unpaired Student's $t$ test (with Welch's correction when variance was unequal) between 2 groups and 1-way ANOVA with post hoc Tukey's test among 3 groups. Non-normally distributed data were analyzed by 2-tailed Mann-Whitney $U$ test between 2 groups and Kruskal-Wallis $H$ test with post hoc Dunn's test among 3 groups. Data with more than 1 variable were evaluated by 2-way repeated-measures ANOVA with post hoc Holm-Š́dák or Tukey's multiple-comparisons tests. Data are shown as mean \pm SEM. All results were considered statistically significant at $P<0.05$.

\section{Study approval}

The protocols for animal experiments described in this paper were approved by the Institutional Animal Care and Use Committees of Boston University School of Medicine and the University of Virginia.

\section{Author contributions}

YW, SS, YY, ZK, MS, KO, MAE, AK, HO, KDM, KH, MAZ, EMY, and JJF designed and performed experiments. SS, KKH, EMP, and $\mathrm{KW}$ directed the research and prepared the manuscript.

\section{Acknowledgments}

This work was funded by NIH grants HL131006, HL132564, and HL138014 to KW, by American Heart Association Postdoctoral Fellowship 17POST33670076 and the Kanae Foundation for the Promotion of Medical Science to SS, and by R01 DK119394 to EMP. JJF is supported by the Ramón y Cajal program of the Spanish Ministerio de Ciencia, Innovación y Universidades (RYC-2016-20026). The CNIC is supported by the Instituto de Salud Carlos III; the Ministerio de Ciencia, Innovación y Universidades; and the Pro CNIC Foundation, and is a Severo Ochoa Center of Excellence (SEV-2015-0505).

Address correspondence to: Kenneth Walsh, Robert M. Berne Cardiovascular Research Center, 415 Lane Road, Suite 1010, PO Box 801394, Charlottesville, Virginia, USA. Phone: 434.243.8303; Email: kw9ar@virginia.edu. 
1. Shlush LI. Age-related clonal hematopoiesis. Blood. 2018;131(5):496-504.

2. Steensma DP, et al. Clonal hematopoiesis of indeterminate potential and its distinction from myelodysplastic syndromes. Blood. 2015;126(1):9-16

3. Fuster JJ, et al. Clonal hematopoiesis associated with TET2 deficiency accelerates atherosclerosis development in mice. Science. 2017;355(6327):842-847.

4. Sano S, Oshima K, Wang Y, Katanasaka Y, Sano M, Walsh K. CRISPR-mediated gene editing to assess the roles of Tet2 and Dnmt3a in clonal hematopoiesis and cardiovascular disease. Circ Res. 2018;123(3):335-341.

5. Sano S, et al. Tet2-mediated clonal hematopoiesis accelerates heart failure through a mechanism involving the IL-1 $\beta / N L R P 3$ inflammasome. J Am Coll Cardiol. 2018;71(8):875-886.

6. Sano S, et al. $J A K 2^{617 F}$-mediated clonal hematopoiesis accelerates pathological remodeling in murine heart failure. JACC Basic Transl Sci. 2019;4(6):684-697.

7. Jaiswal S, et al. Clonal hematopoiesis and risk of atherosclerotic cardiovascular disease. N Engl J Med. 2017;377(2):111-121.

8. Bick AG et al. Inherited causes of clonal hematopoiesis of indeterminate potential in TOPMed whole genomes [preprint]. https://doi.org/10.1101/782748. Posted on bioRxiv September 27, 2019.

9. Bick AG, et al. Genetic interleukin 6 signaling deficiency attenuates cardiovascular risk in clonal hematopoiesis. Circulation. 2020;141(2):124-131.

10. Zink F, et al. Clonal hematopoiesis, with and without candidate driver mutations, is common in the elderly. Blood. 2017;130(6):742-752.

11. Loh PR, et al. Insights into clonal haematopoiesis from 8,342 mosaic chromosomal alterations. Nature. 2018;559(7714):350-355.

12. Genovese G, et al. Clonal hematopoiesis and blood-cancer risk inferred from blood DNA sequence. N Engl J Med. 2014;371(26):2477-2487.

13. Jaiswal S, et al. Age-related clonal hematopoiesis associated with adverse outcomes. N Engl J Med. 2014;371(26):2488-2498.

14. Mas-Peiro S, et al. Clonal haematopoiesis in patients with degenerative aortic valve stenosis undergoing transcatheter aortic valve implantation. Eur Heart J. 2020;41(8):933-939.

15. Dorsheimer L, et al. Association of mutations contributing to clonal hematopoiesis with prognosis in chronic ischemic heart failure. JAMA Cardiol. 2019;4(1):25-33.

16. Fuster JJ, Walsh K. Somatic mutations and clonal hematopoiesis: unexpected potential new drivers of age-related cardiovascular disease. Circ Res. 2018;122(3):523-532.

17. Sano S, Wang Y, Walsh K. Clonal hematopoiesis and its impact on cardiovascular disease. Circ J. 2018;83(1):2-11.

18. Sasi SP, et al. Different sequences of fractionated low-dose proton and single iron-radiation-induced divergent biological responses in the heart. Radiat Res. 2017;188(2):191-203.

19. Severe N, et al. Stress-induced changes in bone marrow stromal cell populations revealed through single-cell protein expression mapping. Cell Stem Cell. 2019;25(4):570-583.e7.

20. Lu R, Czechowicz A, Seita J, Jiang D, Weissman IL. Clonal-level lineage commitment pathways of hematopoietic stem cells in vivo. Proc Natl Acad Sci US A. 2019;116(4):1447-1456.

21. Quesenberry PJ, et al. Engraftment of normal murine marrow into nonmyeloablated host mice. Blood Cells. 1994;20(2-3):348-350.

22. Shimoto M, Sugiyama T, Nagasawa T. Numerous niches for hematopoietic stem cells remain empty during homeostasis. Blood. 2017;129(15):2124-2131.

23. Cabezas-Wallscheid N, et al. Identification of regulatory networks in HSCs and their immediate progeny via integrated proteome, transcriptome, and DNA methylome analysis. Cell Stem Cell. 2014;15(4):507-522.

24. Pietras EM, et al. Functionally distinct subsets of lineage-biased multipotent progenitors control blood production in normal and regenerative conditions. Cell Stem Cell. 2015;17(1):35-46.

25. Moran-Crusio K, et al. Tet2 loss leads to increased hematopoietic stem cell self-renewal and myeloid transformation. Cancer Cell. 2011;20(1):11-24.

26. Laurenti E, et al. Hematopoietic stem cell function and survival depend on c-Myc and N-Myc activity. Cell Stem Cell. 2008;3(6):611-624.

27. Epelman S, et al. Embryonic and adult-derived resident cardiac macrophages are maintained through distinct mechanisms at steady state and during inflammation. Immunity. 2014;40(1):91-104.

28. Chakarov S, et al. Two distinct interstitial macrophage populations coexist across tissues in specific subtissular niches. Science. 2019;363(6432):eaau0964.

29. Boyle AJ, et al. Cardiomyopathy of aging in the mammalian heart is characterized by myocardial hypertrophy, fibrosis and a predisposition towards cardiomyocyte apoptosis and autophagy. Exp Gerontol. 2011;46(7):549-559.

30. Rodriguez-Fraticelli AE, et al. Clonal analysis of lineage fate in native haematopoiesis. Nature. 2018;553(7687):212-216.

31. Sun J, et al. Clonal dynamics of native haematopoiesis. Nature. 2014;514(7522):322-327.

32. Dick SA, et al. Self-renewing resident cardiac macrophages limit adverse remodeling following myocardial infarction. Nat Immunol. 2019;20(1):29-39.

33. Bajpai G, et al. Tissue resident CCR2- and CCR2+ cardiac macrophages differentially orchestrate monocyte recruitment and fate specification following myocardial injury. Circ Res. 2019;124(2):263-278.

34. Cai Z, et al. Inhibition of inflammatory signaling in tet 2 mutant preleukemic cells mitigates stress-induced abnormalities and clonal hematopoiesis. Cell Stem Cell. 2018;23(6):833-849.e5.

35. Rhee AJ, Lavine KJ. New approaches to target inflammation in heart failure: harnessing insights from studies of immune cell diversity. Annu Rev Physiol. 2020;82:1-20.

36. Bajpai G, et al. The human heart contains distinct macrophage subsets with divergent origins and functions. Nat Med. 2018;24(8):1234-1245.

37. Molawi K, et al. Progressive replacement of embryo-derived cardiac macrophages with age. J Exp Med. 2014;211(11):2151-2158.

38. Ito S, et al. Tet proteins can convert 5-methylcytosine to 5-formylcytosine and 5-carboxylcytosine. Science. 2011;333(6047):1300-1303.

39. Ko M, et al. Impaired hydroxylation of 5-methylcytosine in myeloid cancers with mutant TET2. Nature. 2010;468(7325):839-843

40. Svensson E et al. TET2-driven clonal hematopoiesis predicts enhanced response to canakinumab in the CANTOS trial: an 
exploraroty analysis. Circulation. 2018;138(suppl 1):A15111.

41. De Schepper S, et al. Self-maintaining gut macrophages are essential for intestinal homeostasis. Cell. 2018;175(2):400-415.e13

42. Robertson NA, et al. Age-related clonal haemopoiesis is associated with increased epigenetic age. Curr Biol. 2019;29(16):R786-R787.

43. Dai DF, Chen T, Johnson SC, Szeto H, Rabinovitch PS. Cardiac aging: from molecular mechanisms to significance in human health and disease. Antioxid Redox Signal. 2012;16(12):1492-1526.

44. Buscarlet M, et al. Lineage restriction analyses in CHIP indicate myeloid bias for TET2 and multipotent stem cell origin for DNMT3A. Blood. 2018;132(3):277-280

45. Mercier FE, Sykes DB, Scadden DT. Single targeted exon mutation creates a true congenic mouse for competitive hematopoietic stem cell transplantation: the C57BL/6-CD45.1(STEM) mouse. Stem Cell Reports. 2016;6(6):985-992.

46. Franceschi C, Campisi J. Chronic inflammation (inflammaging) and its potential contribution to age-associated diseases. J Gerontol A Biol Sci Med Sci. 2014;69 Supp1 1:S4-S9.

47. Campisi J, Kapahi P, Lithgow GJ, Melov S, Newman JC, Verdin E. From discoveries in ageing research to therapeutics for healthy ageing. Nature. 2019;571(7764):183-192.

48. Wang Y, et al. Wnt5a-mediated neutrophil recruitment has an obligatory role in pressure overload-induced cardiac dysfunction. Circulation. 2019;140(6):487-499.

49. Bray NL, Pimentel H, Melsted P, Pachter L. Near-optimal probabilistic RNA-Seq quantification. Nat Biotechnol. 2016;34(5):525-527.

50. Liao Y, Wang J, Jaehnig EJ, Shi Z, Zhang B. WebGestalt 2019: gene set analysis toolkit with revamped UIs and APIs. Nucleic Acids Res. 2019;47(W1):W199-W205.

51. Shibata R, et al. Adiponectin protects against myocardial ischemia-reperfusion injury through AMPK- and COX-2-dependent mechanisms. Nat Med. 2005;11(10):1096-1103.

52. Shiojima I, et al. Disruption of coordinated cardiac hypertrophy and angiogenesis contributes to the transition to heart failure. J Clin Invest. 2005;115(8):2108-2118.

53. Maruyama S, et al. Follistatin-like 1 promotes cardiac fibroblast activation and protects the heart from rupture. EMBO Mol Med. 2016;8(8):949-966.

54. Nakamura K, et al. Secreted frizzled-related protein 5 diminishes cardiac inflammation and protects the heart from ischemia/ reperfusion injury. J Biol Chem. 2016;291(6):2566-2575. 